\title{
Experimental Analysis of a Wideband Adaptive-MIMO Antenna
}

Michael A. Jensen

jensen@byu.edu

Follow this and additional works at: https://scholarsarchive.byu.edu/facpub

Part of the Electrical and Computer Engineering Commons

\section{Original Publication Citation}

Pinchera, D., et al. "Experimental Analysis of a Wideband Adaptive-MIMO Antenna." Antennas and Propagation, IEEE Transactions on 56.3 (28): 98-13

\section{BYU ScholarsArchive Citation}

Jensen, Michael A., "Experimental Analysis of a Wideband Adaptive-MIMO Antenna" (2008). Faculty Publications. 200.

https://scholarsarchive.byu.edu/facpub/200 
[6] J. S. Colburn and Y. Rahmat-Samii, "Patch antennas on externally perforated high dielectric constant substrates," IEEE Trans. Antennas Propag., vol. 47, pp. 1785-1794, Dec. 1999.

[7] R. F. J. Broas, D. F. Sievenpiper, and E. Yablonovitch, "A high-impedance ground plane applied to a cell-phone handset geometry," IEEE Trans. Microw. Theory Tech., vol. 49, no. 7, pp. 1262-1265, Jul. 2001.

[8] F. Yang and Y. Rahmat-Samii, "Mutual coupling reduction of microstrip antennas using electromagnetic band-gap structure," in Proc. IEEE AP-S Int. Symp. Dig., Jul. 2001, vol. 2, pp. 478-481.

[9] R. Gonzalo, P. de Maagt, and M. Sorolla, "Enhanced patch-antenna performance by suppressing surface waves using photonic-bandgap substrates," IEEE Trans. Microw. Theory Tech., vol. 47, no. 11, pp. 2131-2138, Nov. 1999.

[10] Y. J. Park, A. Herschlein, and W. Wiesbeck, "A photonic bandgap (PBG) structure for guiding and suppressing surface waves in millimeter-wave antennas," IEEE Trans. Microw. Theory Tech., vol. 49, no. 10, pp. 1854-1859, Oct. 2001.

[11] F. Yang and Y. Rahmat-Samii, "Microstrip antennas integrated with electromagnetic band-gap (EBG) structures: A low mutual coupling design for array applications," IEEE Trans. Antennas Propag., vol. 51, pp. 2939-2949, Oct. 2003.

[12] M. F. Abedin and M. Ali, "Effects of a smaller unit cell planar EBG structure on the mutual coupling of a printed dipole array," IEEE Antennas Wireless Propag. Lett., vol. 4, pp. 274-276, 2005.

[13] F. Yang and Y. Rahmat-Samii, "A low profile circularly polarized curl antenna over electromagnetic band-gap (EBG) surface," Microw. Opt. Tech. Lett., vol. 31, no. 4, pp. 478-481, Nov. 2001.

[14] F. Yang and Y. Rahmat-Samii, "Reflection phase characterizations of the EBG ground plane for low profile wire antenna applications," IEEE Trans. Antennas Propag., vol. 51, no. 10, pp. 2691-2703, Oct. 2003.

[15] M. F. Abedin and M. Ali, "Effects of EBG reflection phase profiles on the input impedance and bandwidth of ultra-thin directional dipoles," IEEE Trans. Antennas Propag., vol. 53, no. 11, pp. 3664-3672, Nov. 2005.

[16] M. F. Abedin and M. Ali, "A low profile dipole antenna backed by a planar EBG structure," in Proc. IEEE Int. Workshop on Antenna Tech., Small Antennas and Novel Metamaterials, Mar. 6-8, 2006, pp. 13-16.

[17] Z. Li and Y. Rahmat-Samii, "PBG, PMC and PEC ground planes: A case study of dipole antennas," in Proc. IEEE AP-S Int. Symp. Dig., Jul. 2000, vol. 2, pp. 674-677.

[18] D. Sievenpiper, J. Schaffner, B. Loo, G. Tangonan, R. Harold, J. Pikulski, and R. Garcia, "Electronic beam steering using a varactortuned impedance surface," in Proc. IEEE AP-S Int. Symp. Dig., Jul. 2001, vol. 1, pp. 174-177.

[19] D. Sievenpiper, L. Zhang, R. F. J. Broas, N. G. Alexopolous, and E. Yablonovitch, "High impedance electromagnetic surfaces with a forbidden frequency band," IEEE Trans. Microw. Theory Tech., vol. 47, no. 11, pp. 2059-2074, Nov. 1999.

[20] Y. J. Lee, J. Yeo, R. Mittra, and W. S. Park, "Application of electromagnetic bandgap (EBG) superstrates with controllable defects for a class of patch antennas as spatial angular filters," IEEE Trans. Antennas Propag., vol. 53, no. 1, pp. 224-235, Jan. 2005.

[21] H. Mosallaei and K. Sarabandi, "A compact wide-band EBG structure utilizing embedded resonant circuits," IEEE Antennas Wireless Propag. Lett., vol. 4, pp. 5-8, 2005.

[22] J. M. Bell and M. F. Iskander, "A low-profile archimedian spiral antenna using an EBG ground plane," IEEE Antennas Wireless Propag. Lett., vol. 3, pp. 223-226, 2004.

[23] S. Tse, B. S. Isquierdo, J. C. Bachelor, and R. J. Langley, "Reduced sized cells for electromagnetic bandgap structures," Electron. Lett., vol. 39, no. 24, pp. 1699-1701, Nov. 2003.

[24] O. Folayan and R. J. Langley, "Wideband reduced size electromagnetic bandgap structure," Electron. Lett., vol. 41, no. 20, pp. 1099-1100, Sep. 2005.

[25] S. Shahparnia and O. M. Ramahi, "Electromagnetic interference (EMI) reduction from printed circuit boards (PCB) using electromagnetic bandgap structures," IEEE Trans. Electromagn. Compat., vol. 46, pp. 580-587, Nov. 2004.

[26] I. J. Bahl and P. Bhartia, Microwave Solid State Circuit Design. New York: Wiley, 1988.

\section{Experimental Analysis of a Wideband Adaptive-MIMO Antenna}

Daniele Pinchera, Jon W. Wallace, Marco Donald Migliore, and Michael A. Jensen

Abstract-An experimental measurement campaign to investigate the wideband performance of an adaptive multiple-input multiple-output (AdaM) antenna consisting of two active and six parasitic antenna elements. The measurements are conducted over a $30 \mathrm{MHz}$ bandwidth at a center frequency of $2.55 \mathrm{GHz}$ and in a variety of locations in an indoor environment. The system uses a traditional two-element array at the transmitter and either a two-element traditional array or the AdaM antenna at the receiver. The results show that compared to the traditional array, the AdaM receive antenna can achieve the same throughput with an average power reduction of $2.7 \mathrm{~dB}$ and a maximum power reduction of 7.7 dB. The analysis further shows that the parasitic configuration works well over the entire frequency band, although improved power reduction can be obtained when the operation bandwidth is limited.

Index Terms-Broadband communication, multiple-input multipleoutput (MIMO) systems, parasitic antennas.

\section{INTRODUCTION}

Multiple-input multiple-output (MIMO) technology [1], [2] shows considerable promise as an approach for increasing the spectral efficiency of wireless communication. Because of this potential, significant research effort has been devoted to this topic, resulting in remarkably rapid evolution of the technology into emerging commercial standards and products.

As the technology transitions from the research laboratory to commercial products, efforts are focused on finding ways to effectively implement MIMO communication using simple, robust, and inexpensive system components which can fit within constrained volumes. Efforts to reduce the number of required transmit or receive electronic sub-systems (which can be expensive in terms of cost and battery power) have demonstrated that antenna selection algorithms can provide high performance under many circumstances [3], although implementation of this concept requires the addition of high-performance microwave switches and effective compensation techniques for the changing impedance of the driven elements due to coupling with adjacent elements for compact devices.

Recently solutions based on reconfigurable antenna systems have been proposed [4]-[8] which provide power gains comparable those offered by antenna selection but with a reduced set of transmit or receive electronic chains. This kind of antenna, specifically designed to work in MIMO communication systems, will thus be referred to as "MIMO antennas." In particular in [8] antenna pattern reconfiguration is achieved by adapting the loads on passive antenna elements adjacent to the driven elements. To date, this adaptive MIMO (AdaM) antenna structure has been analyzed only for a limited set of narrowband measurements.

Manuscript received December 31, 2006; revised November 5, 2007. This work was supported in part by the Italian Ministry of University (MIUR) under a Program for the Development of Research of National Interest (PRIN Grant \#2007APSKNA_001).

D. Pinchera and M. D. Migliore are with the DAEIMI, University of Cassino, 03043 Cassino, Italy (e-mail: mdmiglio@unicas.it). USA.

M. A. Jensen is with ECEN, Brigham Young University, Provo, UT 84602 J. W. Wallace is with Jacobs University Bremen, 28759 Bremen, Germany. Digital Object Identifier 10.1109/TAP.2008.917008 
The goal of this paper is to more carefully explore the potential performance of the AdaM antenna in a set of realistic environments and over wideband operation, i.e., when the channel coherence bandwidth is smaller than the signal bandwidth, leading to a frequency selective channel. The study is accomplished by construction of a candidate AdaM antenna architecture and characterization of its performance in several indoor settings using a wideband MIMO channel sounder.

\section{SYSTEM MODELING AND MEASUREMENT}

Roughly speaking in an AdaM antenna the switching of the parasitic elements changes the position and the level of the lobes of the radiating patterns, thus changing the MIMO channel matrix. The representation given in [8] allows us to model the MIMO channel matrix $\mathbf{H}(f)$, incorporating the impact of the electromagnetically coupled parasitic elements for a fixed frequency $f$. To characterize a wideband system, we can sub-divide the band of interest in $K$ smaller sub-bands (similar to what is done in OFDM MIMO systems [9]), so that the system response in each sub-band can be considered constant ("flat"), with the system response determined at the sub-band center frequency $f_{k}$. The channel capacity $C_{k}$ of the deterministic MIMO channel, for the $k$ th sub-band, is then

$$
C_{k}=\sum_{i=1}^{r_{k}} \log _{2}\left(1+\frac{P_{T} \Sigma_{i, k} \alpha_{i, k}}{\sigma_{\eta, k}^{2}}\right), \quad[\mathrm{bit} / \mathrm{s} / \mathrm{Hz}]
$$

where $P_{T}$ is the total transmitted power, $\Sigma_{i, k}$ is the square of the $i$ th singular value [11] of the matrix $\mathbf{H}\left(f_{k}\right), r_{k}$ is the rank of $\mathbf{H}\left(f_{k}\right), \sigma_{\eta, k}^{2}$ is the variance of the additive white Gaussian noise at the receiver in the $k$ th sub-band, and $\alpha_{i, k}$ is the fraction of the total transmitted power $P_{T}$ allocated to the $i$ th spatial channel of the $k$ th sub-band, chosen according to the waterfilling algorithm [2]. So the overall maximum throughput of the MIMO channel for the wideband system becomes

$$
R_{\max }=\Delta f \sum_{k=1}^{K} C_{k}, \quad[\mathrm{bit} / \mathrm{s}]
$$

where $\Delta f$ is the bandwidth of each sub-band.

The singular values depend on the loads on which the passive antennas are terminated. Consequently the channel seen by the active elements depends on the loads. From a communication point of view, the AdaM antenna allows to obtain a set of potential communication channels, among which the most advantageous in terms of channel capacity is chosen. From an electromagnetic point of view, the use of parasitic antennas terminated on controllable loads allows a larger set of possible patterns compared to a classic MIMO antenna (without passive elements) giving further degrees of freedom in the maximization of the bit rate. The beamforming can be considered as a spatial processing of the electromagnetic signal, performed before the classic MIMO signal data processing. It involves complex interactions of the signals in the close near field of the active antennas. Consequently, generally the pattern of the antenna are very complex, since they are non linear functions of the load impedances and of the frequency, apart from some simple cases like LOS environments, and are of little or no usefulness to analyze the antenna performance. For this reason the pattern of the antenna will not be reported in this paper, and we will follow a system approach, analyzing the singular values of the communication channel matrix.

To assess the practical performance of the AdaM antenna using experimental measurements in a variety of environments we have built a prototype of the AdaM antenna array, shown in Fig. 1, which has been designed and fabricated to operate in the $2.4 \mathrm{GHz}$ ISM band. The antenna consists of eight wire antennas on a ground plane (monopoles) resonating at the center frequency of $2.55 \mathrm{GHz}$ (chosen in order to avoid interference with an existing wireless local area network). The

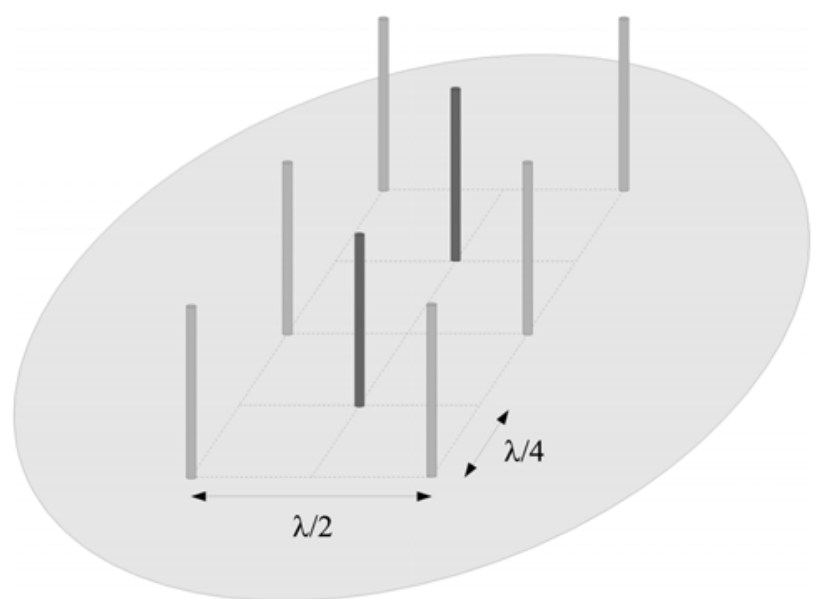

Fig. 1. Parasitic MIMO antenna: dark gray—active elements; light gray—parasitic elements.

two inner and six outer wires represent the active and parasitic elements, respectively.

The reflection coefficients of the monopoles in absence of the other monopoles is lower than $-20 \mathrm{~dB}$ at the center frequency. The reflection coefficients of the two active monopoles of the AdaM antenna depends on the load configurations. However, in our approach the channel capacity takes into account also the losses due to the mismatch of the active antennas.

The controllable terminations attached to the parasitic elements are based on a Philips BAP63 PIN diode. Each switch accepts two different driving voltage states: $0 \mathrm{~V}$ (OFF state) or $5 \mathrm{~V}$ (ON state). A vector network analyzer was used to measure the impedance of each switch in both states, with the mean impedance values at the center frequency being $Z_{\mathrm{OFF}}=61.3+j 13.7 \Omega$ and $Z_{\mathrm{ON}}=12.1+j 2.9 \Omega$. These PIN diode switches can change states in less than $2 \mu \mathrm{s}$.

Fair evaluation of the gain enabled by use of an AdaM antenna requires comparison of the measurement results with those obtained when a traditional array is used. Therefore, reference measurements use a two-element array of monopole antennas with half-wavelength spacing. This array is identical to the AdaM array with the parasitic elements removed.

Experimental assessment of the potential performance of a MIMO system equipped with wideband AdaM antennas requires a suite of sophisticated MIMO channel measurements in which the parasitic loads are switched over all possible combinations. In this work, this is accomplished using simple modifications to a MIMO channel sounder which typically uses switches to connect different antennas to a single transmitter or receiver. The complete description of the channel sounder and of its calibration can be found in [12], [13].

To use this sounder to characterize the channel when the AdaM antenna is used, two of the switch control signals are used to sequentially connect the active antenna elements to the RF electronics. The remaining switch control signals are used to cycle through all possible combinations of switch states on the AdaM parasitic elements for each connected active antenna. In this way, a (wideband) channel matrix is obtained for each possible parasitic load combination, allowing determination of the optimal switch combination from the perspective of capacity.

\section{EXPERIMENTAL RESULTS}

The system is configured by attaching the traditional two-element monopole array to the transmitter (TX) and either an identical traditional monopole array or the AdaM array at the receiver (RX). The 
measurement of the wideband $2 \times 2$ MIMO channel uses a multitone signal consisting of 30 tones at $1 \mathrm{MHz}$ spacing with a center frequency of $2.55 \mathrm{GHz}$. This frequency spacing is sufficient given that the measured correlation bandwidth is approximately $5 \mathrm{MHz}$. Each measurement therefore produces $302 \times 2$ channel matrices (one for each tone). In order to estimate the maximum capacity obtainable on the measured channel we use water-filling [2] to determine the power allocation coefficients $\alpha_{i, k}$ which indicate the fraction of the total transmit power $P_{T}$ assigned to the $i$ th spatial channel in the $k$ th frequency bin ( $1 \leq i \leq 2$ and $1 \leq k \leq 30$ ). This computation is accomplished by applying the water-filling algorithm to the $60 \times 60$ block diagonal matrix formed from the $302 \times 2$ channel matrices. Effectively, the algorithm considers all available space-frequency sub-channels as power is allocated to maximize the achievable system mutual information. It is important to emphasize that the capacity computed in this fashion is appropriate for a single parasitic state, and therefore a single channel snapshot requires 64 such measurements (six parasitic elements each with two possible states). Measurement of this entire data set requires less than $20 \mathrm{~ms}$ of time on the channel probing system. The stability of the channel over a time window larger than $20 \mathrm{~ms}$ was verified for all measurements by comparing two successive channel snapshots to ensure that their difference was negligible.

Once we have acquired the channel matrices for the reference antenna and the 64 different termination combinations for the AdaM antenna, we must effectively compare the relative performance of these different systems. To do this, we compute the value of $P_{T} / \sigma_{\eta}^{2}$ in (1) required to achieve a value of $R_{\max }=300 \mathrm{Mbit} / \mathrm{s}$ in (2) for each configuration, where we have assumed that the noise power is equal across the bandwidth so that $\sigma_{\eta, k}=\sigma_{\eta}$ for all $k$. The value of this ratio for each AdaM configuration relative to that for the reference antenna system is then used as a metric for evaluation. If a particular configuration can achieve the specified rate with lower transmit power $P_{T}$, then this is an effective gain to the system. Numerically, we evaluate the ratio

$$
\Delta P_{T}=\frac{\left(P_{T} / \sigma_{\eta}^{2}\right)_{\mathrm{AdaM}}}{\left(P_{T} / \sigma_{\eta}^{2}\right)_{\text {reference }}}
$$

where the subscripts "AdaM" and "reference" indicate the antenna configuration used. It is necessary to underline that to achieve a system throughput close to the waterfilling channel capacity the transmitter needs to know the state of the channel, and the realization of a proper feedback from the receiver to transmitter that can be an issue; anyway, since in this paper we are interested only in benchmarking the performances of the wideband AdaM antenna we will neglect this issue.

The experimental campaign consists of measurements taken in the Clyde Engineering Building on the Brigham Young University campus. The measurements are grouped into sweeps obtained by moving the receiver in one-inch steps (about $\lambda / 5$ which is approximately equal to the measured correlation distance) along a straight line while the transmitter remains stationary. Fig. 2 shows the plan for the fifth floor of the building which is the site for the majority of the measurements, where the positions for the transmitter and receiver for the $i$ th measurement sweep are designated as $\mathrm{TX}_{i}$ and $\mathrm{RX}_{i}$, respectively. Sweep 1 consists of 18 measurements, while each of the remaining 7 sweeps consists of 68 measurements. It should be noted that for sweep 8 , the receiver is placed on the fourth floor of the building at the relative position shown in Fig. 2.

\section{A. Power Reduction}

Fig. 3 plots the ratio $\Delta P_{T}$ defined in (3) for all measured channels. The average power reduction obtained using the AdaM antenna is $2.7 \mathrm{~dB}$. This is significant, as it indicates that the optimally loaded parasitic elements enable communication at the same rate with almost half

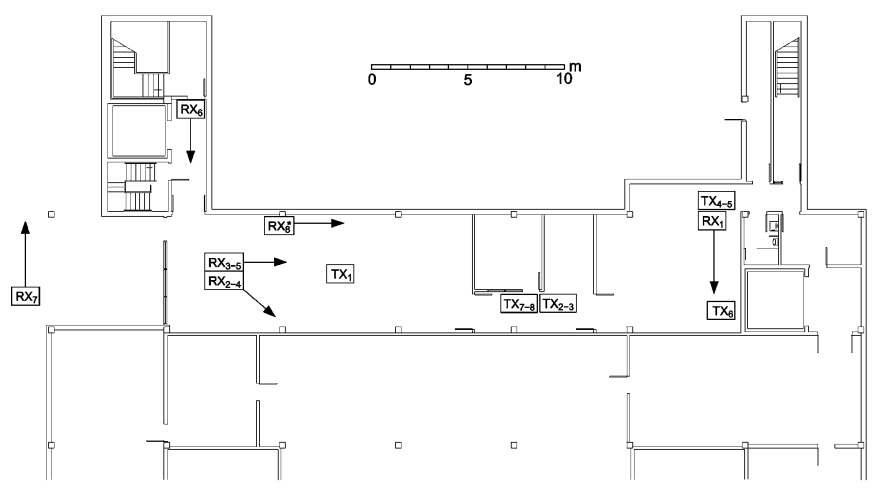

Fig. 2. Plan of the fifth floor of the Clyde Engineering building. The labels describe the position and direction of movement of the receiving and transmitting antennas in the 8 sweeps. For sweep number 8 , the receiver was on the fourth floor.

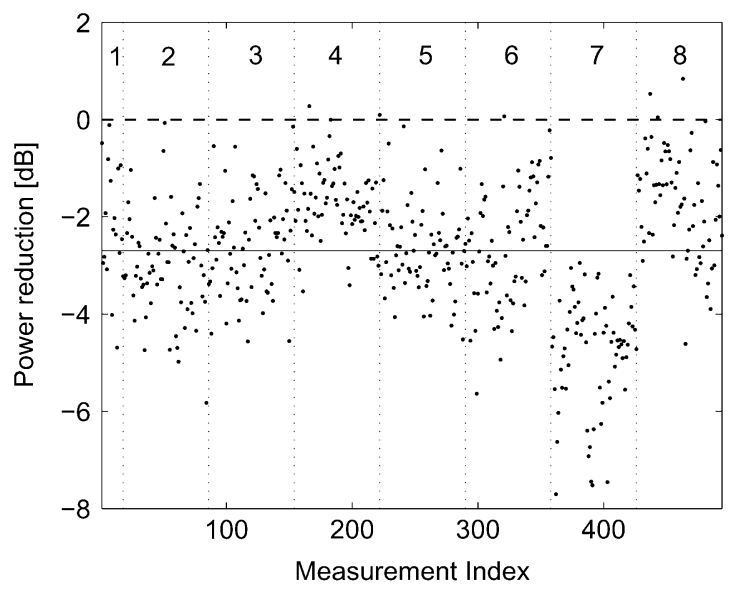

Fig. 3. The power reduction $\Delta P_{T}$ to obtain the same target $R_{\max }$ for all measured cases. The vertical dashed lines delineate the 8 measurement sweeps, while the horizontal solid line represents the average power reduction.

the power. When the receiver is placed on a terrace (sweep 7), there are no scatterers in the vicinity of the receiver resulting in reduced angle spread at this location. Therefore, the AdaM antenna can have a significant impact on the performance (largest power reduction). When the transmitter and receiver are on different floors (sweep 8), a condition leading to rich multipath, the AdaM antenna has little impact on performance.

Fig. 4 shows a histogram of the data shown Fig. 3 along with the best fitting Gaussian distribution (mean -2.7, standard deviation 1.4).

\section{B. Space-Frequency Sub-Channels}

Examination of the singular values of the full $60 \times 60$ channel matrix provides some insight into the beneficial impact of the AdaM antenna system. Fig. 5 plots these singular values for a channel that achieves the "average" power reduction of $2.7 \mathrm{~dB}$ both with the AdaM (using the best loading combination) and reference receive antennas. The 60 singular values represent the two singular values (corresponding to spatial channels) for the channel matrix at each of the 30 frequencies (frequency channels). It is worth noting that in scenarios with limited multipath angular spread, the second (smaller) spatial singular value of each channel matrix may be smaller than the first singular value of all other matrices. In this case, there will be a discontinuity between the 30th and 31st singular values, as seen in Fig. 5.

The results in Fig. 5 show that the AdaM RX antenna increases the singular values in general which accounts for part of the power reduc- 


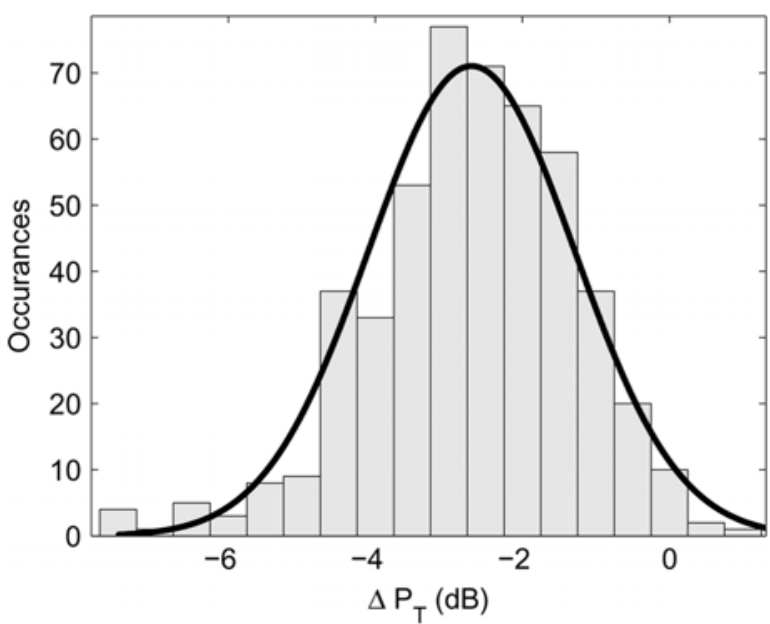

Fig. 4. The distribution of the ratio $\left(\left(P_{T} / \sigma_{n}^{2}\right)_{\mathrm{AdaM}} /\left(P_{T} / \sigma_{n}^{2}\right)_{\text {sole active }}\right)$ to obtain the same target capacity.

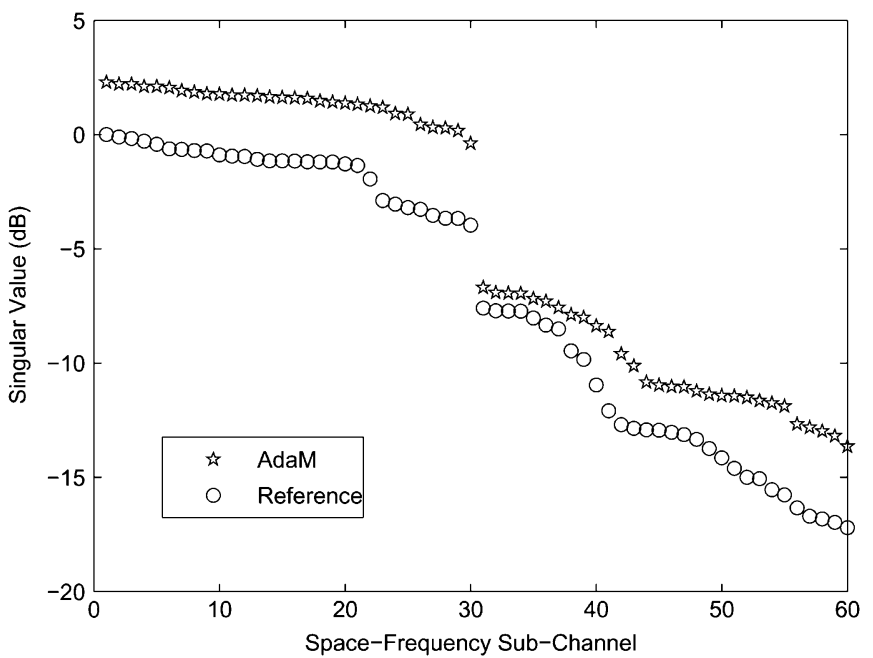

Fig. 5. Singular values for a channel matrix that achieves the "average" power reduction.

tion, making the singular value distribution flatter. This allows to obtain a higher throughput, since all the SISO sub-channels associated to the singular values convey almost the same quantity of information [14]. This point is perhaps more clearly demonstrated by plotting the coefficients $\alpha_{i, k}$ which represent the fraction of the transmitted power on each sub-channel as allocated by the water-filling solution. Fig. 6 plots these coefficients for the channel used in Fig. 5. The algorithm allocates the power more evenly across the 60 sub-channels, since the coefficient distribution in Fig. 6 is closer to a uniform distribution. This means that each sub-channel contribute to the overall channel capacity almost in the same way, or in other words that all the sub-channels are equally "good" to convey information. Because these power allocation coefficients directly incorporate the impact of the channel singular values, for the remainder of this discussion we will focus on these coefficients.

We can also use this analysis approach to explore the behavior of the AdaM antenna relative to the reference array in other types of channels. For example, we consider the absolute "worst" channel depicted in Fig. 3 for which the AdaM antenna requires an increase in transmit power of $\Delta P_{T}=0.8 \mathrm{~dB}$ to achieve the target rate. Fig. 7 plots the power allocation coefficients for this case, where we now see that the reference antenna has more uniform power distribution across the sub-channels. It is clear that in this environment, the adaptivity

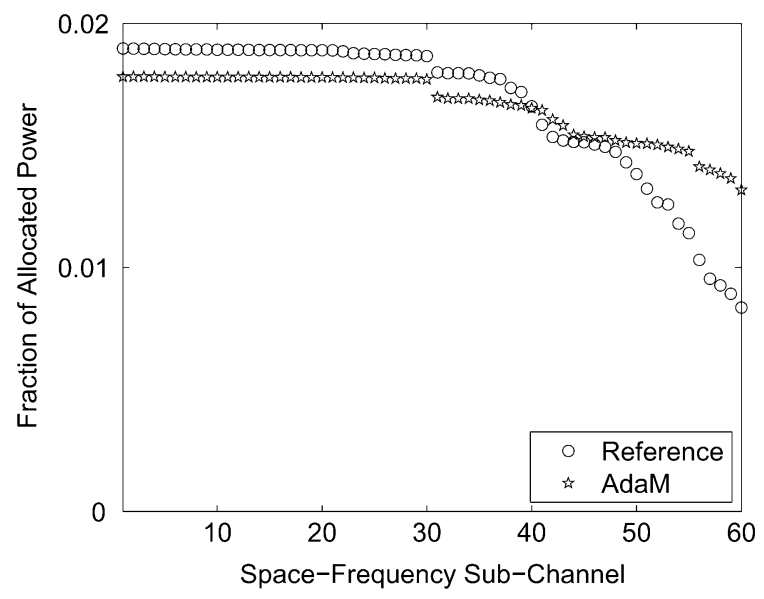

Fig. 6. Power allocation coefficients for the "average" case.

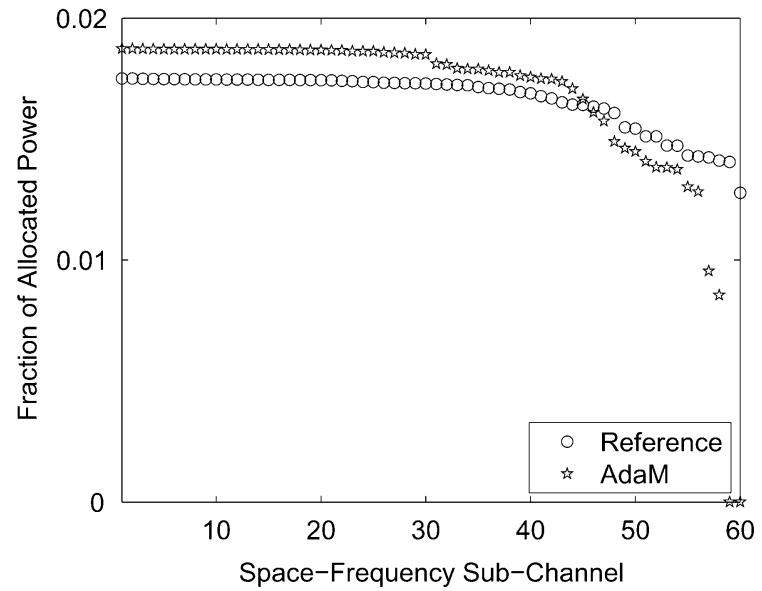

Fig. 7. Power allocation coefficients for the channel matrix that achieves the "worst" power reduction.

enabled by the parasitic elements is incapable of improving on the performance, presumably because the space-frequency response of the reference array is already well suited for sampling the incident multipath field. However such cases are rare in this campaign, with the AdaM antenna requiring more power than the reference antenna in only 6 out of 494 measured channels.

In contrast, Fig. 8 shows the power allocation coefficients for the channel in which the AdaM antenna gives the largest power reduction (7.7 dB). In this case, the reference antenna only provides one strong spatial channel for each frequency while the AdaM antenna enables the effective use of both spatial channels over the entire band. We emphasize that this case occurs when the receiver is on an outside terrace which is well removed from most of the scatterers in the propagation environment, resulting in a small angular spread of the multipath components incident on the receiver. The AdaM antenna therefore appears able to adapt the array response to effectively sample the incident field and achieve much better performance than can be obtained by the reference structure. It is important to emphasize that the larger aperture and two-dimensional array geometry of the AdaM antenna gives it a beamforming advantage over the reference array which is particularly effective for channels with narrow angular spread such as this one. In fact, detailed numerical studies reveal that the performance gained by the larger aperture alone is negligible for most environments with wider angular spread and that the bulk of the benefit generally comes from the adaptivity of the array. Since such environments are rare in the indoor 


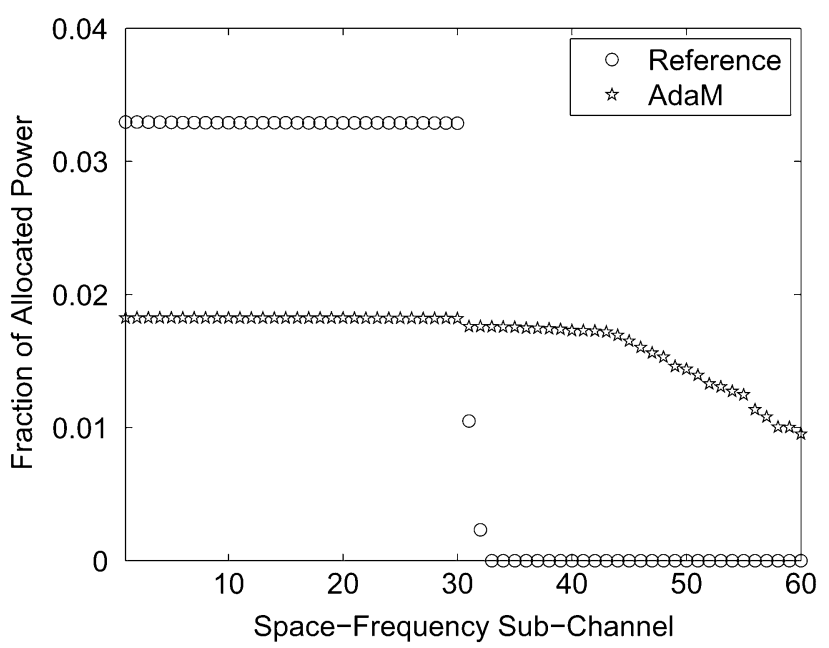

Fig. 8. Power allocation coefficients for the channel matrix that achieves the "best" power reduction.

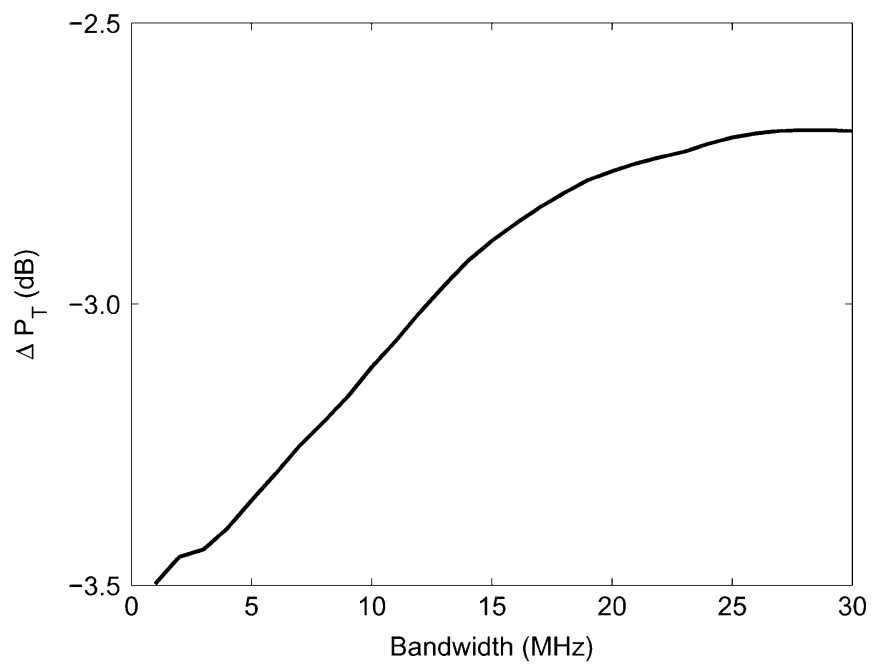

Fig. 9. Power reduction achieved by the AdaM antenna as a function of the operation bandwidth.

scenarios in which the measurements were performed (only sweep 7 demonstrates this behavior), the increased aperture accounts for only a small part of the average benefit offered by the AdaM antenna.

It should also be mentioned that large power reduction is also achieved when the transmitter and receiver are relatively closely spaced (see sweeps 2 and 3 in Fig. 3), reinforcing the idea that the adaptive capability of the parasitic elements can be used effectively in environments with a strong line-of-sight component or multipath with narrow angular spread.

\section{Bandwidth Considerations}

It is important to emphasize that the optimal parasitic loading achieves a power reduction by improving the spatial and frequency response of the antenna. For example, careful examination of the singular values (or power allocation coefficients) discussed in Section III-B shows that the optimal load achieves an equalization of the singular values in frequency (i.e., singular values remain more constant over the operation bandwidth). It is intuitive, however, that this effect will degrade as the operation bandwidth increases.

To study this effect, consider Fig. 9 which shows the power reduction averaged over all measurements achieved by the AdaM antenna relative to the reference antenna as a function of the operation bandwidth. When the bandwidth is narrow, the beamforming achieved by the optimal loading of the AdaM antenna achieves a power reduction of $3.5 \mathrm{~dB}$. As the bandwidth is increased, the loading may not be as effective over the entire band, resulting in the observed decrease in power reduction. So this result suggests that the use of loads whose frequency response can be modified could improve the performances of this kind of antennas when the bandwidth is large.

It is important to point out, however, that the degradation in power reduction is only $0.8 \mathrm{~dB}$ as the bandwidth is increased from $1 \mathrm{MHz}$ to $30 \mathrm{MHz}$, indicating that the optimal loading works well over reasonable operation bandwidths. This is an important consideration for practical implementation of AdaM antennas.

\section{CONCLUSION}

This paper reports on the experimental investigation of the wideband performance of AdaM antennas in a wideband MIMO communication system. The measurement campaign uses a $2 \times 2$ MIMO system with a traditional array at the transmitter and either a traditional (reference) or AdaM array at the receiver. The data consists of measurements taken in a variety of locations in an indoor environment. Summarizing the experimental results, for a $1 \mathrm{MHz}$ channel bandwidth, the AdaM antenna can achieve the same rate as the reference antenna with an average transmit power reduction of $3.5 \mathrm{~dB}$. As the bandwidth is increased to $30 \mathrm{MHz}$, the AdaM antenna still achieves an average transmit power reduction of $2.7 \mathrm{~dB}$. The study also show that in environments characterized by multipaths with narrow angle spread, the AdaM antenna provides particular benefit. This is due to the fact that the beamforming provided by the parasitic elements' switching acts as a spatial processing performed before the terminals of the active elements of the antenna, and hence modifies the communication channel itself.

We emphasize that these results have been obtained without optimization of the antenna and switch structure, and therefore further improvement may be possible through an improved antenna design. It is also noteworthy that in a frequency selective channel, the AdaM antenna adapts both its spatial and frequency response to optimize performance, in contrast to operation in a frequency flat channel where only the spatial response is important. This observation suggests that the use of loads with a controllable frequency behavior should result in improved performance due to increase flexibility in adapting the overall array frequency response. Finally, since this study has considered only an AdaM antenna at one end of the link, the results in [8] suggest that additional performance gain can be achieved through the use of AdaM arrays at both transmit and receive.

\section{REFERENCES}

[1] "Special issue on gigabit wireless," Proc. IEEE, vol. 92, no. 2, Feb. 2004.

[2] G. J. Foschini and M. J. Gans, "On limits of wireless communications in a fading environment when using multiple antennas," Wireless Personal Commun., vol. 6, pp. 311-335, Mar. 1998.

[3] A. F. Molisch, M. Z. Win, and J. H. Winters, "Capacity of MIMO systems with antenna selection," in Proc. IEEE Int. Conf. Communications, 2001, vol. 2, pp. 570-574.

[4] B. A. Cetiner et al., "Multifunctional reconfigurable MEMS integrated antennas for adaptive MIMO systems," IEEE Commun. Mag., pp. 62-70, Dec. 2004.

[5] D. Piazza and K. R. Dandekar, "Reconfigurable antenna solution for MIMO-OFDM systems," Electron. Lett., vol. 42, no. 8, Apr. 2006.

[6] B. A. Cetiner, E. Akay, E. Sengul, and E. Ayanoglu, "A MIMO system with multifunctional reconfigurable antennas," IEEE Antennas Wireless Commun. Lett., vol. 5, pp. 463-466, 2006.

[7] M. Mowlér and B. Lindmark, "Reconfigurable MEMS antennas for wireless applications," presented at the Eur. Conf. Antennas and Propagation, EuCAP 2006, Nice, France, Nov. 6-10, 2006. 
[8] M. D. Migliore, D. Pinchera, and F. Schettino, "Improving channel capacity using adaptive MIMO antennas," IEEE Trans. Antennas Propag., vol. 54, no. 11, pt. 2, pp. 3481-3489, Nov. 2006.

[9] R. S. Blum, Y. G. Li, J. H. Winters, and Q. Yan, "Improved space-time coding for MIMO-OFDM wireless communications," IEEE Trans. Commun., vol. 49, no. 11, pp. 1873-1878, Nov. 2001.

[10] C. E. Shannon, "A mathematical theory of communication," Bell Syst. Tech. J., no. 27, pp. 379-423, 623-656, 1948.

[11] G. H. Golub and C. F. Van Loan, Matrix Computations. Baltimore, MD: The John Hopkins Univ. Press, 1996.

[12] B. T. Maharaj, L. P. Linde1, J. W. Wallace, and M. A. Jensen, "A cost-effective wideband MIMO channel sounder and initial co-located $2.4 \mathrm{GHz}$ and $5.2 \mathrm{GHz}$ measurements," in Proc. IEEE Int. Conf. Acoustics, Speech, and Signal Processing (ICASSP), Philadelphia, PA, Mar. 18-23, 2005, vol. 3, pp. 981-984

[13] J. W. Wallace and M. A. Jensen, "Time-varying MIMO channels: Measurement, analysis, and modeling," IEEE Trans. Antennas Propag., vol. 54, no. 11, pt. 1, pp. 3265-3273, Nov. 2006.

[14] A. Paulraj, R. Nabar, and D. Gore, Introduction to Space-Time Wireless Communications. Cambridge, U.K.: Cambridge Univ. Press, 2003.

\section{A Bandwidth Estimation Approach for the Asymptotic Waveform Evaluation Technique}

Zhen Peng and Xin-Qing Sheng

\begin{abstract}
The asymptotic waveform evaluation (AWE) technique has been widely used for handling the fast parameter sweep. However, how to determine the sweep bandwidth is still an open question. This work proposes an efficient and flexible bandwidth estimation approach. This approach also naturally leads to an efficient multipoint AWE technique. The validity of the proposed approach is verified by numerical experiments on the AWE-incorporated hybrid finite element/boundary integral (FE/BI) method for scattering problem. Numerical experiments show that this approach can greatly improve the efficiency and practical capability of the AWE technique.
\end{abstract}

Index Terms-Asymptotic waveform evaluation (AWE), bandwidth estimation, rational approximation.

\section{INTRODUCTION}

In many practical applications in electromagnetic simulation, it is desired to pursue a wide region of information by varying one or two parameter. For scattering analysis, our interest often includes a wide band of frequencies. For microwave imaging, it is necessary to determine radar cross section over a wide range of both frequencies and incident angles to rebuild the microwave imaging of the targets. These problems are usually categorized as the fast parameter sweep problem.

Manuscript received April 24, 2007; revised September 3, 2007. This work was supported by the 973 Program under Grant 2005CB321702.

Z. Peng is with the Graduate University of Chinese Academy of Sciences, Beijing 100080, China, and also with the Institute of Electronics, Chinese Academy of Sciences, Beijing 100080, China, and also with the Center for Electromagnetic Simulation, School of Information Science and Technology, Beijing Institute of Technology, Beijing 100081, China (e-mail: zhenpeng2008@hotmail.com).

X.-Q. Sheng is with the Center for Electromagnetic Simulation, School of Information Science and Technology, Beijing Institute of Technology, Beijing 100081, China (e-mail: xsheng@ @it.edu.cn).

Color versions of one or more of the figures in this paper are available online at http://ieeexplore.iee.org.

Digital Object Identifier 10.1109/TAP.2008.917017

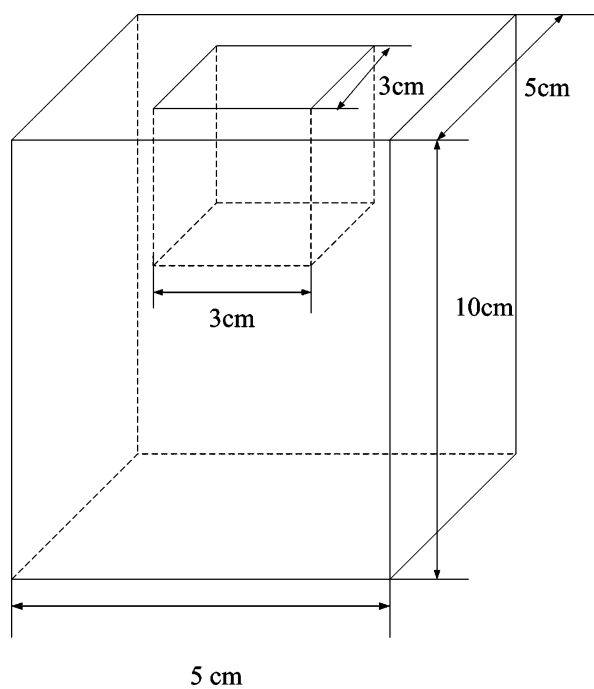

Fig. 1. Conducting brick target with an empty cavity.

The previous works show that the rational approximation technique is a kind of efficient way of handling the above problem. This technique first constructs a rational function of the desired parameter, and then utilizes the rational function to approximate the parameter over a region. There are different ways to construct the rational function. Among them, the asymptotic waveform evaluation (AWE) is a general and efficient one, and has been widely used. This technique was originally developed for high-speed circuit analysis [1], and then was applied to the finite element and finite difference analysis of electromagnetic problem [2]-[4]. In [5], [6], the AWE has also been applied to the moment of method for solving scattering problem. Recently, the multipoint Galerkin AWE (MGAWE) and the well-conditioned AWE (WCAWE) have been developed in [7], [8]. In sum, most of previous works lay stress on either the application of AWE to different problems or how to achieve a wider bandwidth of the available expansion band. There is a very important issue needed to be investigated, which is how to determine the bandwidth of the available expansion band for a desired accuracy. A bandwidth estimation approach was presented in [9] and successfully applied to the FEM fast frequency sweep. In this paper, a different and flexible approach is proposed for the efficient and accurate estimation of the bandwidth.

In this paper, the AWE-incorporated FE/BI method is first presented for the fast frequency and angle sweep of the radar cross section (RCS). Then a flexible bandwidth estimation approach is proposed for the AWE technique. Finally, Numerical experiments demonstrate the validity of the bandwidth estimation approach for the AWE-incorporated FE/BI method.

\section{Application of AWE to Hybrid FE/BI Method}

It is known that the problem of scattering by a composite object can be discretized by using hybrid FE/BI method [10] as

$$
\left[\begin{array}{ccc}
\boldsymbol{K}_{I I} & \boldsymbol{K}_{I S} & 0 \\
\boldsymbol{K}_{S I} & \boldsymbol{K}_{S S} & B \\
\mathbf{0} & \boldsymbol{P} & \boldsymbol{Q}
\end{array}\right]\left\{\begin{array}{l}
\boldsymbol{E}_{I} \\
\boldsymbol{E}_{S} \\
\boldsymbol{H}_{S}
\end{array}\right\}=\left\{\begin{array}{l}
0 \\
0 \\
b
\end{array}\right\}
$$

where $\left[\boldsymbol{K}_{I I}\right],\left[\boldsymbol{K}_{S S}\right],\left[\boldsymbol{K}_{I S}\right],\left[\boldsymbol{K}_{S I}\right],[\boldsymbol{B}]$ are sparse FEM matrixes, $[\boldsymbol{P}]$ and $[\boldsymbol{Q}]$ are dense MoM matrixes, and $\left[\boldsymbol{K}_{I I}\right],\left[\boldsymbol{K}_{S S}\right]$ are usually symmetric, $[\boldsymbol{B}]$ is skew symmetric. Rewrite (1) as

$$
\boldsymbol{Z}(k) \boldsymbol{I}(k ; \theta, \phi)=\boldsymbol{V}(k ; \theta, \phi)
$$

